\title{
203 A MEMBRANE-TETHERED IL-15/IL-15 RECEPTOR FUSION PROTEIN ENHANCES THE PERSISTENCE AND EFFICACY OF CD70-TARGETED TRUC-T CELLS
}

Jian Ding*, Lindsay Webb, Troy Patterson, Michelle Fleury, Adam Zieba, Holly Horton, Robert Hofmeister, Dario Gutierrez, Robert Tighe. TCR2 Therapeutics, Cambridge, MA, USA

Background Adoptive cell therapies have shown great promise in hematological malignancies. To realize the potential of $\mathrm{T}$ cell therapies in solid tumors, we have developed $\mathrm{T}$ cell receptor fusion construct $\left(\mathrm{TRuC}^{\circledR}\right) \mathrm{T}$ cells, which are equipped with an engineered $\mathrm{T}$ cell receptor that utilizes all TCR signaling subunits and recognizes tumor-associated antigens independent of HLA. Previously, we have described the discovery and preclinical efficacy of fratricide-resistant TRuC-T cells targeting CD70, a tumor antigen overexpressed in various solid and hematological malignancies. As a strategy to enhance $\mathrm{T}$ cell effector function and persistence in the hostile tumor microenvironment, we engineered anti-CD70 TRuC-T cells to coexpress a membrane-bound IL15Ra-IL15 fusion protein (IL$15 \mathrm{fu})$. IL-15 is a common ? chain cytokine that promotes the differentiation, maintenance, and effector function of memory $\mathrm{CD} 8+\mathrm{T}$ cell subsets and confers resistance to IL-2-mediated activation induced cell death (AICD).

Methods $\mathrm{T}$ cells were activated by $\mathrm{CD} 3 / \mathrm{CD} 28$ stimulation and lentivirally transduced with a T2A-containing bicistronic vector encoding the anti-CD70 CD3?-TRuC and the IL-15fu proteins; the cells were further expanded for 9 days in media containing IL-7/IL-15. Surface co-expression of the TRuC and IL$15 \mathrm{fu}$ proteins and the $\mathrm{T}$ cell memory phenotype was assessed by flow cytometry. In vitro persistence was tested in a repeated stimulation assay in which $\mathrm{T}$ cells were challenged by addition of fresh $\mathrm{CD} 70+$ target cells every four days with longitudinal assessment of T-cell expansion, phenotype, cytokine production, and cytotoxicity. In vivo, the antitumor efficacy of the anti-CD70 TRuC/IL-15fu T cells was evaluated in MHC class I/II deficient NSG mice bearing human tumor xenografts.

Results The anti-CD70 TRuC and IL-15fu proteins showed high transduction efficiency and robust co-expression on the surface of $\mathrm{T}$ cells. The IL- $15 \mathrm{fu}$ significantly increased the proportion of naïve cells within the TRuC-T cell product, most dramatically in the CD8 + subset. In vitro, TRuC-T cells bearing the IL-15fu showed greatly enhanced expansion and persistence upon repeated stimulation with $\mathrm{CD} 70+$ target cells. Moreover, the IL-15fu enhanced T-cell survival and persistence under unstimulated, cytokine-free conditions. In vivo, the antitumor activity of CD70-targeted TRuC-T cells was significantly improved by IL- $15 \mathrm{fu}$ in multiple tumor models and was associated with enhanced intratumoral T-cell accumulation and a preferential expansion of CD8 $+\mathrm{T}$ cells.

Conclusions The addition of the IL- $15 \mathrm{fu}$ improved the phenotype, persistence, and anti-tumor activity of CD70-targeted TRuC-T cells, potentially increasing the likelihood of clinical benefit in patients with CD70 overexpressing solid and liquid cancers.

Ethics Approval All animal studies were conducted by TCR2 Therapeutics staff at the Charles River Laboratories CRADL facility under a protocol approved by the Charles River Laboratories Institutional Animal Care and Use Committee.

http://dx.doi.org/10.1136/jitc-2021-SITC2021.203 